\title{
SYNTHESIS OF NOVEL HETEROCYCLIC QUINOLONE COMPOUND FOR ANTI -TUBERCULAR ACTIVITY
}

\author{
Rahul K. Godge ${ }^{1}$, Rahul Kunkulol ${ }^{2}$ \\ ${ }^{1}$ Pravara Rural College of Pharmacy, \\ ${ }^{2}$ Pravara Institute of Medical Sciences, Loni BK - Rahata, Ahmednagar, 413736 (M.S.). India .
}

\section{ABSTRACT}

In last few decades, though significant progress has been made in the treatment and control strategies of tubercular infections by introducing new diagnostic and monitoring tools and combination therapy, it still continues to be severe problem. The need of study was only because of there are many drugs in market to treat infection but most of the drugs are showing resistance because of the same it is difficult to treat the infection. In this study we chosen quinolone nucleus for study and over it. Thus with the aim of developing novel molecule with improved potency for treating Mycobacterium tuberculosis H37Rv strain infections and with decreased probability of developing drug resistance. Methodology: The synthesis of Quinolone derivatives, starting from substituted aniline and ethyl acetoacetate, by conventional organic reaction and results of investigations of their anti-mycobacterial activity. Results: MICs of the synthesized compounds are compared with existing drugs Cytotoxicity. The substituted quinolones are synthesized by taking mixture of 7-substituted-2-(3-chloro-2-oxopropyl) quinolin-4(1H)-one and different secondary amines. Many compounds have shown promising activity while some were inactive. Conclusion: It was found that Compound $A_{1}, A_{3}, B_{1}, B_{3}$, have shown promising anti tubercular activity whereas compound $A_{2}, A_{4}, B_{2}, B_{4}$ were showing moderate anti tubercular activity against std. Streptomycin.

KEYWORDS: Quinolone derivative; Well diffusion method; Spectral analysis; Elemental analysis.

\section{INTRODUCTION}

Microbial infections remain the major cause of death over the world. Emergence of multi-drug resistant to different infectious organisms like $M$. tuberculosis made the condition most alarming.[1-2] Tuberculosis, MTB, or TB is a deadly infectious disease caused by various strains of mycobacteria; usually Mycobacterium tuberculosis. According to World Health Organization (WHO) TB is a global pandemic, which has become an important world-wide public health menace with one-third of the world's population infected by the TB bacillus. Most infections do not have symptoms, known as latent tuberculosis and about one in ten latent infections eventually progresses to active disease which, if left untreated, kills more than $50 \%$ of those so infected. People with weak immune systems (those with HIV/AIDS, those receiving immunosuppressive

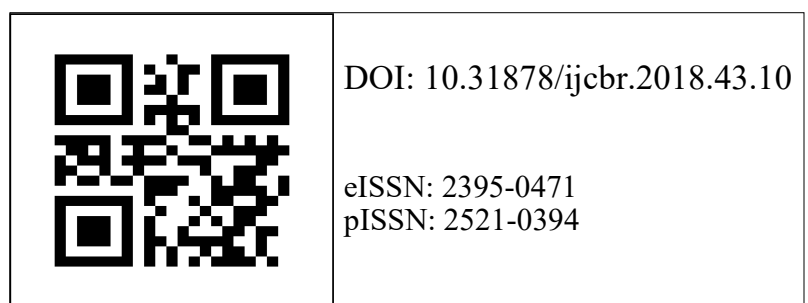

drugs and chemotherapy) are at a greater risk for developing TB disease. There is currently a growing concern about the progress and spread of multidrug and extensively drug resistant tuberculosis (MDR/XDR -TB), which has the potential to paralyze TB care schemes. The focal theme of this thesis is the exploration of new strategies in the field of modern drug discovery for the development of new drugs, which are capable of overcoming MDR/XDR-TB. The present work was aimed to synthesized new compound and evaluate it for antituberculer activity. Therefore, there is an urgent demand for a new class of antimicrobial agent with a different mode of action and it led medicinal chemists to explore a wide variety of chemical structures.

Quinoline was first isolated from coal tar in 1834, it was also recognized as pyrolytic degradation product of cinchonamine, an alkaloid closely related to quinine[36 ]. The name quinoline was derived from quina, a Spanish version of a local South American name for the bark of quinine-containing cinchona Species. Several Synthetic anti-malarial drugs are based on the quinoline nucleus, Chloroquine is an example. Several antibiotics like fluro-quinolones now in clinical use were 4quinolone-based antibiotics.[7-10] Quinoline is a color-

Correspondence: Mr. Rahul Keshav Godge, Pravara Rural College of Pharmacy, Loni BK, Tal- Rahata, Dist: Ahmednagar, Maharashtra-413736, India. E-mail: rahul godge@rediffmail.com 
less liquid of bp. $237^{\circ} \mathrm{C}$. It turns yellow on standing and has pyridine like smell.Miscible with most organic solvents and dissolves in water to about $0.7 \%$ at room temperature. Slightly weaker base $\left(\mathrm{pK}_{\mathrm{a}}=4.94\right)$ than pyridine $\left(\mathrm{pK}_{\mathrm{a}}=5.2\right)$. It reacts with acid to yield salts which are sparing soluble in water[11-12].

Compounds containing quinolones exhibiting variety of pharmacological and biological activities[13-25].

\section{Experimental}

General: The nucleus and its derivatives were analyzed by different ways. The melting points were recorded on electrothermal apparatus and are uncorrected.(IR) spectra were determined on Bruker IFS-66 FTIR (Bruker Bioscience, USA) using $\mathrm{KBr}$ pallets and wave number (í) was reported in $\mathrm{cm}-1 .{ }^{1} \mathrm{H}$ NMR spectra on a Bruker Avance $300 \mathrm{MHz}$ instrument using DMSO as solvent using TMS as internal standard; the chemical shifts $(\delta)$ were reported in ppm with coupling constants $(J)$ are given in $\mathrm{Hz}$. Signal multiplicities were represented by $\mathrm{s}$ (singlet), d (doublet), t (triplet), ds (double singlet), dd (double doublet), $\mathrm{m}$ (multiplet) and bs (broad singlet. Elemental analysis was performed on a Hera- cus CHNRapid Analyzer. Analysis indicated by the symbols of the elements of functions was within $\pm 0.4 \%$ of the theoretical values. The purity of the compounds was checked on silica gel coated Al plates (Merck).

\section{Synthesis of 2-methyl-7-substituted-4-quinolone}

A mixture of $(0.01 \mathrm{~mol})$ substituted anilines and $(0.01$ mol) ethyl acetoacetate was stirred, was heated on oil bath at $180^{\circ} \mathrm{C}$ for two hours, The crude solid was filtered, dried and recrystallized from ethanol .

\section{Synthesis of 7-substituted-2-(3-chloro-2-oxopropyl) quinolin-4(1H)-one (II)}

An quantity of 0.01 moles of I was dissolved in $25 \mathrm{ml}$ of glacial acetic acid. If did not dissolve completely, the mixture was slightly warmed. The solution was cooled in ice bath with stirring. To this chloroacetyl chloride (0.12 mole) solution added drop wise. To prevent the occurrence of vigorous reaction the temp was maintained at $0^{\circ} \mathrm{C}$ then reaction was heated for 30 mins after this cool the mixture and pour over crushed ice white product was separated by filtration. The product was washed with $50 \%$ aqueous acetic acid and finally with water. It was recrystallized.

\section{Synthesis of derivatives of substituted 4-quinolones III}

A mixture of 0.01 mole of each II were taken in dry 250 $\mathrm{ml}$ round bottom flask separately to this distilled alcohol is added as solvent and to this different secondary amines were added in 0.01 mole concentrations and refluxed for 2 hour after reflux add reaction mixture to crushed ice precipitation formed is filtered and recrys- tallized.

\section{Spectral Data}

$\mathrm{A}_{1}-\mathrm{IR}(\mathrm{KBr}): 3487.07$ (-N-H str), 3032.16 (Ar-H str), 2841 ( CH str), 1721 (-C=O str), 1685 (CO str amide), 1160 (-C-N str), 801(-C-Clstr) NMR: d8.5-8.(0 1H, S$\mathrm{NH})$, 7.91-7.87 (4H , m-Quinolone), 7.37-6.97 4H,m -Ar$\mathrm{CH} 3.81-3.122 \mathrm{H}, d-\mathrm{CH}_{2}$

$\mathrm{A}_{2}$ - IR (KBr): 3487.07 (-N-H str), 3135(Ar-H str), 2561 ( CHstr), 1721 (-C=O str), 1685 (CO str amide), 1353 (-SO2NH2 str), 1200 (-C-N str) 801(-C-Clstr)

$\mathrm{A}_{3}-\mathrm{IR}(\mathrm{KBr}): 3372$ (-N-H str), 3032(Ar-H str), 2850 ( CHstr), 1720 (-C=O str), 1685 (CO str amide), 1200 (-C-N str) 780 (-C-Clstr)

$\mathrm{A}_{4}-\mathrm{IR}(\mathrm{KBr}): 3420$ (-OH str), 3380 (-N-H str), 3020(Ar-H str), 2862 ( CHstr), 1700 (-C=O str), 1685 (CO str amide), 1230 (-C-N str) 759 (-C-Clstr)

$\mathrm{B}_{1}-\mathrm{IR}(\mathrm{KBr}): 3367$ (-NH str), 3021 (-Ar-H str), 2848(-CH str), 1728 ( - $\mathrm{C}=\mathrm{O}$ str), 1655 (CO str amide), 1157 (-C-N str) 662 (-C-Br str)

$\mathrm{B}_{2}-\mathrm{IR}(\mathrm{KBr}): 3367$ (-NH str), 3021 (-Ar-H str), 2848(-CH str), 1728 ( $-\mathrm{C}=\mathrm{O}$ str), 1655 (CO str amide), 1353 ((-SO2NH2 str))1157 (-C-N str) 662 (-C-Br str)

$\mathrm{B}_{3}-\mathrm{IR}(\mathrm{KBr}): 3372$ (-N-H str), 3032(Ar-H str), 2850 ( CHstr), 1720 (-C=O str), 1685 (CO str amide), 1200 (-C-N str) 645 (-C-BR str)

$\mathrm{B}_{4^{-}}$IR $(\mathrm{KBr}): 3420$ (-OH str), 3370 (-N-H str), 3020(Ar-H str), 2850 ( CHstr), 1690 (-C=O str), 1680 (CO str amide), 1230 (-C-N str) 650 (-C-Br str)

Anti-tubercular activity: The compounds were tested in-vitro for their anti-tubercular activity against $\mathrm{H}_{37} \mathrm{Rv}$ Strain.

Method: Alamar Blue Dye: The anti-tubercular screening was carried out by Middle brook 7H9 agar medium against $\mathrm{H}_{37} \mathrm{Rv}$ Strain. Middle brook 7H9 agar medium was inoculated with Mycobacterium tuberculosis of $\mathrm{H}_{37} \mathrm{Rv}$ Strain. The inoculated bottles were incubated for $37^{\circ} \mathrm{C}$ for 4 weeks. At the end of 4 weeks they were checked for growth.

\section{RESULT}

Scheme: $\left(A_{1}-A_{4}, B_{1}-B_{4}\right)$

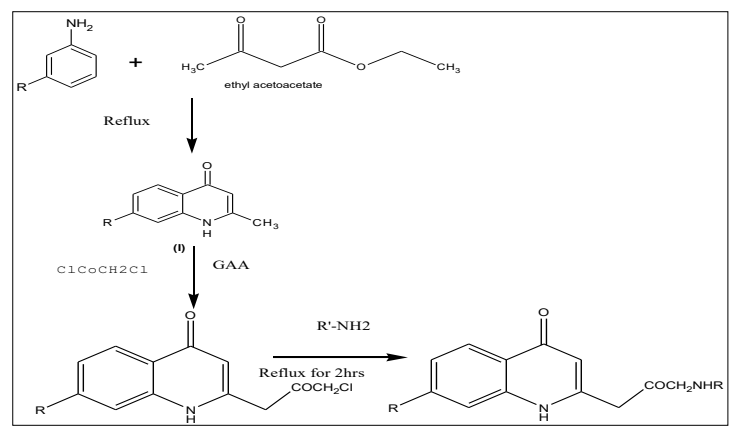


Table 1. Derivative compounds

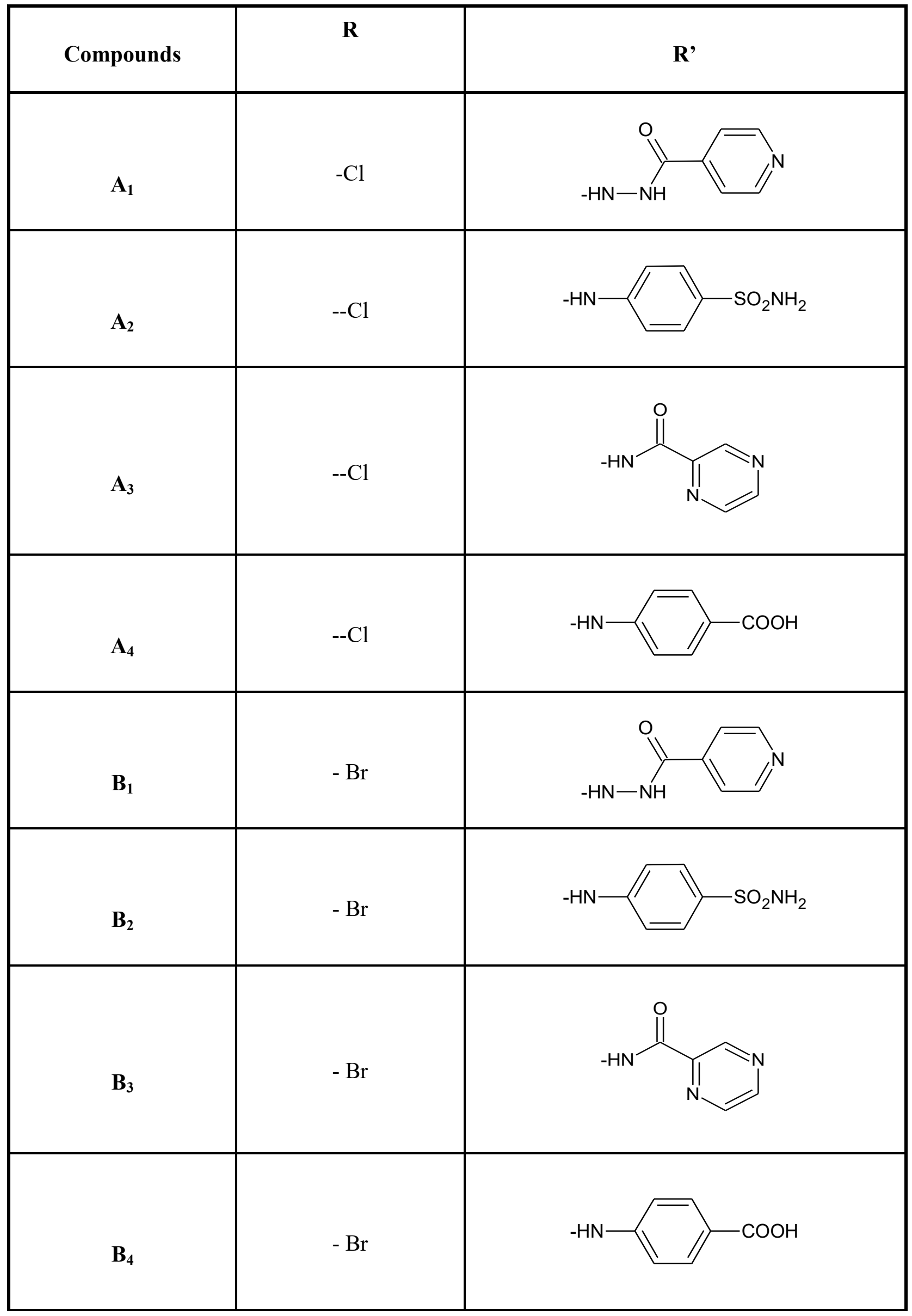


Table 2. Anti-tubercular activity of substituted 4-quinolones compounds

\begin{tabular}{|l|l|l|l|l|l|l|l|l|l|l|}
\hline $\begin{array}{l}\text { Comp } \\
\text { ID }\end{array}$ & $\mathbf{1 0 0}$ & $\mathbf{5 0}$ & $\mathbf{2 5}$ & $\mathbf{1 2 . 5}$ & $\mathbf{6 . 2 5}$ & $\mathbf{3 . 1 2 5}$ & $\mathbf{1 . 6}$ & $\mathbf{0 . 8}$ & $\mathbf{0 . 4}$ & $\mathbf{0 . 2}$ \\
\hline & & & & & & & & & & \\
\hline A1 & S & S & S & S & S & S & S & R & R & R \\
\hline A2 & S & S & S & S & R & R & R & R & R & R \\
\hline A3 & S & S & S & S & S & S & S & S & R & R \\
\hline A4 & S & S & S & R & R & R & R & R & R & R \\
\hline B1 & S & S & S & S & S & S & S & R & R & R \\
\hline B2 & S & S & S & S & S & R & R & R & R & R \\
\hline B3 & S & S & S & S & S & S & S & S & S & R \\
\hline B4 & S & S & S & S & R & R & R & R & R & R \\
\hline $\begin{array}{l}\text { Strepto- } \\
\text { mycin }\end{array}$ & S & S & S & S & S & R & R & R & R & R \\
\hline
\end{tabular}

Table 3. Analytical data of the compound

\begin{tabular}{|c|c|c|c|c|c|c|c|c|}
\hline \multirow{2}{*}{$\begin{array}{l}\text { Comp. } \\
\text { Code }\end{array}$} & \multirow{2}{*}{$\begin{array}{c}\text { Mol. } \\
\text { Formula }\end{array}$} & \multirow{2}{*}{$\begin{array}{l}\text { Mol. } \\
\text { Wt. }\end{array}$} & \multirow{2}{*}{$\begin{array}{l}\text { m.p. } \\
{ }^{\circ} \mathrm{C}\end{array}$} & \multirow{2}{*}{$\begin{array}{c}\text { Yield } \\
\%\end{array}$} & \multirow{2}{*}{$\begin{array}{c}\mathbf{R}_{\mathrm{f}} \text { val- } \\
\text { ue }\end{array}$} & \multicolumn{3}{|c|}{$\begin{array}{c}\text { Elemental analyses } \\
\text { Calcd. (Found) }\end{array}$} \\
\hline & & & & & & C & H & $\mathbf{N}$ \\
\hline$A_{1}$ & $\mathrm{C}_{18} \mathrm{H}_{15} \mathrm{ClN}_{4} \mathrm{O}_{3}$ & 370.78 & $180-182$ & 79.85 & 0.50 & 58.31 & 4.08 & 15.11 \\
\hline$A_{2}$ & $\mathrm{C}_{18} \mathrm{H}_{16} \mathrm{ClN}_{4} \mathrm{O}_{4} \mathrm{~S}$ & 405.85 & $142-144$ & 72.53 & 0.57 & 53.27 & 3.97 & 10.35 \\
\hline$A_{3}$ & $\mathrm{C}_{17} \mathrm{H}_{13} \mathrm{ClN}_{4} \mathrm{O}_{3}$ & 356.76 & $130-132$ & 69.52 & 0.53 & 57.23 & 3.67 & 15.70 \\
\hline$A_{4}$ & $\mathrm{C}_{18} \mathrm{H}_{22} \mathrm{ClN}_{3} \mathrm{O}_{4}$ & 379.83 & $136-138$ & 74.54 & 0.50 & 56.92 & 5.84 & 11.0 \\
\hline$B_{1}$ & $\mathrm{C}_{18} \mathrm{H}_{15} \mathrm{BrN}_{4} \mathrm{O}_{3}$ & 415.24 & $110-112$ & 75.34 & 0.61 & 52.06 & 3.64 & 13.49 \\
\hline $\mathrm{B}_{2}$ & $\mathrm{C}_{18} \mathrm{H}_{16} \mathrm{BrN}_{3} \mathrm{OS}$ & 450.30 & $140-142$ & 74.67 & 0.59 & 48.01 & 3.58 & 9.33 \\
\hline $\mathrm{B}_{3}$ & $\mathrm{C}_{17} \mathrm{H}_{13} \mathrm{BrN}_{4} \mathrm{O}_{3}$ & 401.21 & $112-114$ & 72.23 & 0.65 & 50.89 & 3.27 & 13.9 \\
\hline $\mathrm{B}_{4}$ & $\mathrm{C}_{18} \mathrm{H}_{22} \mathrm{BrN}_{3} \mathrm{O}_{4}$ & 424.28 & $136-138$ & 62.12 & 0.64 & 50.95 & 5.23 & 9.90 \\
\hline
\end{tabular}


Rahul K et al. Synthesis of novel heterocyclic Quinolone compound for anti -tubercular activity.

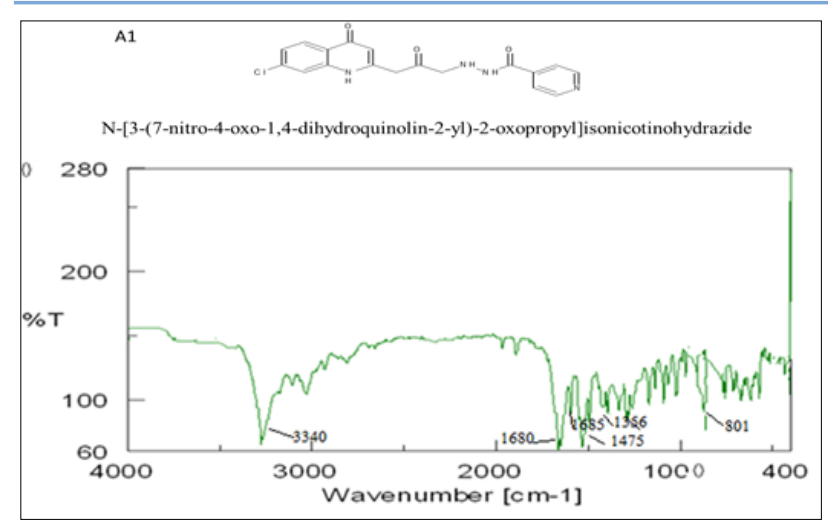

Figure 1. IR Spectra of $A_{1}$

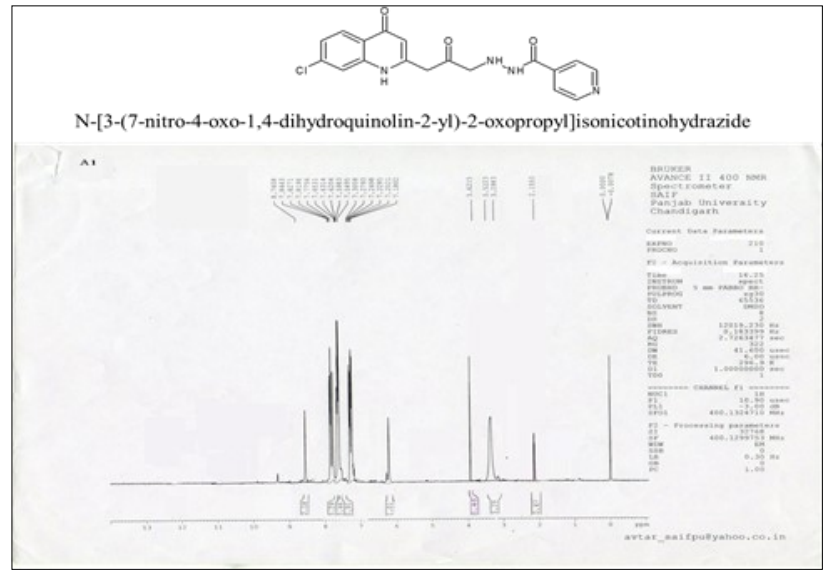

Figure 2. NMR Spectra of $A_{1}$

\section{DISCUSSION:}

In the present research work, we have synthesized 8 new substituted 4-quinolones derivatives as explained in the scheme. The purity of the compounds was checked by TLC and melting point. Structures of these compounds were confirmed by IR, 1 HNMR and elemental analysis. The synthesized compounds were subjected to anti tubercular activity by Alamar Blue Dye method against the standard streptomycin.

Compound $A_{1}, A_{3}, B_{1}, B_{3}$ have shown promising antitubercular activity against streptomycin at concentration of $1.6 \mathrm{mcg} / \mathrm{ml}$ by interpreting data of MIC. With the suitable molecular modification and manipulation with possible SAR studies of these compounds, promising anti tubercular agents can be obtained. [26, 27].

\section{CONCLUSION}

The substituted quinolones are synthesized by taking mixture of 7-substituted-2-(3-chloro-2-oxopropyl) quinolin-4(1H)-one and different secondary amines. It was found that Compound $A_{1}, A_{3}, B_{1}, B_{3}$, have shown promising anti tubercular activity whereas compound $A_{2}$, $A_{4}, B_{2}, B_{4}$ were showing moderate anti tubercular activity against std. Streptomycin.

Acknowledgement: Authors are thankful to Dr. Bhatt
Laboratory for the antitubercular activity data and Punjab university for providing spectral and elemental analysis.

\section{REFERENCES}

1. Ania AE, Idokob J, Dalyopc Y B. Pitmangd SLT. Article title. Roy. Soci. Trup. Med. 2009;103:67.

2. Said HA, Abdel GS, Morlock G, Cooksey RC. Article title Int. J. Infect. Dis.2009;,13, 673.

3. Antimicrobial - Definition from the MerriamWebster Online Dictionary. http://www.merriamwebster.com/dictionary/Antimicrobial. Retrieved 2009-05-02.

4. John HK, John MB. Wilson and Gisvold's Text book of Organic medicinal and Pharmaceutical Chemistry. 11th Edition, Philadelphia: Lippincott Williams and Wilkins; 2004: 299-301.

5. Rang HP, Dale MM, Ritter JM, Moore P K. Pharmacology. $5^{\text {th }}$ Edition, New Delhi: Elsevier India Pvt Ltd; 2003: 667-72.

6. Silverstein SC, Stienman RM, Cahhn ZA. Endocytosis. Ann. Rev. Biochem. 1977; 46: 669-72.

7. Stephonson TJ. Inflammation. In: Underwood JCE. General and Systemic Pathology. 4th Edition, London: Churchill Livingstone. 2004: 201-22.

8. Baxter JD, Rosseau GG. Glucocoticoid hormone action: an overview. Monogram. Endocrinal. 1979; 12:1-24.

9. Vinay Kumar, Cotran RD, Robbins S. Basic Pathology. 6th Edition, Bangalore: Prism Book Pvt Ltd; 1997: 25 $-46$.

10. Hardman JG, Limbird LE. Goodman Gilman's The Pharmacological Basis of Therapeutics. $10^{\text {th }}$ edition, New York: McGraw-Hill Medical Publishing Division; 2001: 687-730.

11. John HK, John MB. Wilson and Gisvold's Text book of Organic medicinal and Pharmaceutical Chemistry. $11^{\text {th }}$ Edition, Philadelphia: Lippincott Williams and Wilkins; 2004: 818-22.

12. Nugteren $\mathrm{DH}$, Hazelhof $\mathrm{E}$. Isolation and properties of intermediates in prostaglandin biosynthesis. Biochem. Biophysics. Acta 1973; 326: 448-461

13. Chun MW, Olmstead KK, Choi YS, Chong OL, ChongKyo L, Joong HK, et al. Synthesis and biological activities of truncated acridone: Structure-activity relationship studies of cytotoxic 8-hydroxy-4-quinolone. Bioorganic \& Medicinal Chemistry Letters. 1997;7 (1): 789-92.

14. Hong C, Kim S, Kim Y. Novel 5-amino-6methylquinolone anti-bacterial: A new class of non- 
6-fluoroquinolones. Bioorganic \& Med. Chemistry Letters 1997;7(14):1875-1878.

15. Srivastava S, Srivastava SK, Shukla A, Chauhan P, Purlet S. Synthesis and the amides of methemoglobin toxicity of $6 / 7$ mono or disubstituted quinolone. Bioorganic \& Med. Chemistry Letters 1999 9: 25-30.

16. Masahiko H, Hiroto K, Tetsushi K, Tomio K, Tomoaki $\mathrm{K}$. Synthesis and anti-HIV activity of arylpiperazinyl fluoroquinolones: a new class of anti-HIV agents. Bioorganic \& Med. Chemistry Letters.1999; 9: 30633068.

17. Fukui $H$, Shibata T, Naito T, Nakano J, Maejima T, Senda $\mathrm{H}$, et al. Synthesis and Antibacterial Activity of Novel 7-(3-Substituted-3 or 4-trifluoromethyl- 1pyrrolidinyl)-8-methoxyfluoroquinolones.

Bioorganic \& Med. Chemistry Letters 1998; 8: 283338.

18. Robert J, Kerns RJ, Michael J, Glenn WR, Vaka F, Richard GR, et al. Piperazinyl-Linked Fluoroquinolone Dimers Possessing Potent Antibacterial Activity Against Drug-Resistant Strains of Staphylococcus aureus. Bioorganic \& Med. Chemistry Letters 2003; 13: $1745-49$.

19. Foroumadi A, Soltani F, Moshafi MH, Ashraf-Askari R. Synthesis and in vitro antibacterial activity of some $\quad \mathrm{N}$-(5-aryl-1,3,4-thiadiazole-2-yl)piperazinyl quinolone derivatives. II. Farmaco 2003; 58: $1023-$ 28.

20. Xia Y, Yang Z, Peng X, Bastow KF, Nakanishi Y, Priya N.. Antitumor Agents. Part 226: Synthesis and Cytotoxicity of 2-Phenyl-4-quinolone Acetic Acids and Their Esters. Bioorganic \& Med. Chemistry Letters 2003; 13: 2891-93.

21. Scott HW, Marianne CTG, Murali D, Shen Z, Pitts WJ, Junqing $G$, et al. Novel inhibitors of IMPDH a highly potent and selective quinolone-based series. Bioorganic \& Med. Chemistry __Letters 2003; 13(3), 543-46.

22. Piyasena $H$, Nathan $C$, Min D, Joanne $T$, Christopher G, Boissard, et al. The synthesis and structureactivity relationships of 3-amino-4-benzylquinolin-2ones: discovery of novel KCNQ2 channel openers. Bioorganic \& Med. Chemistry Letters 2004; 14: 1615 -1618 .

23. Qun L, Woods KW, Wang W, Nan-Horng L, Claiborne A, Wen-zhen G. Design, synthesis, and activity of achiral analogs of 2-quinolones and indoles as nonthiolfarnesyl transferase inhibitors. Bioorganic \& Med. Chemistry Letters 2005; 15: 2033-39.
24. Sriram D, Yogeeswari P, Basha JS, Radhab DR, Nagaraja V. Synthesis and antimycobacterial evaluation of various 7-substituted ciprofloxacin derivatives. Bioorganic \& Medicinal Chem. 2005; 13: 5774-78.

25. Lucero BA, Gomes CR, Frugulhetti IC, Faro LV, Alvarenga L, Maria C. Synthesis and anti-HSV-1 activity of quinolonic acyclovir analogues. Bioorganic \& Med. Chemistry Letters 2006; 11: 1010 -13 .

26. Farhanullah, Yeon Kim S, Yoon EJ, Choi EC, Sunghoon $\mathrm{K}$, et al. Design and synthesis of quinolinones as methionyl-tRNA synthetase inhibitors. Bioorganic \& Medicinal Chem. 2006; 14: 7154-59

27. Sephra N. Rampersad. Multiple Applications of Alamar Blue as an Indicator of metabolic Function and Cellular Health in Cell Viability Bioassays. Sensors (Basel). 2012;12(9): 12347-12360.

How to Cite this article: Rahul K. Godge, Rahul Kunkulol. Synthesis of novel heterocyclic Quinolone compound for anti -tubercular activity. Int. j. clin. biomed. res. 2018;4(3): 44-49. 